\title{
On the Design and Deployment of RFID Assisted Navigation Systems for VANETs
}

\author{
Wei Cheng, Member, IEEE, Xiuzhen Cheng, Senior Member, IEEE, \\ Min Song, Senior Member, IEEE, Biao Chen, Member, IEEE, and Wendy W. Zhao
}

\begin{abstract}
In this paper, we propose a systematic approach to designing and deploying a RFID Assisted Navigation System (RFIDANS) for VANETs. RFID-ANS consists of passive tags deployed on roads to provide navigation information while the RFID readers attached to the center of the vehicle bumper query the tag when passing by to obtain the data for navigation guidance. We analyze the design criteria of RFID-ANS and present the design of the RFID reader in detail to support vehicles at high speeds. We also jointly consider the scheduling of the read attempts and the deployment of RFID tags based on the navigation requirements to support seamless navigations. The estimation of the vehicle position and its accuracy are also investigated.
\end{abstract}

Index Terms-RFID assisted navigation systems, vehicle networks, GPS, system design.

\section{INTRODUCTION}

$\mathrm{R}$ ADIO Frequency Identification (RFID) has attracted considerable attentions in recent years for its broad applications in ubiquitous computing. In this paper, we propose a RFID Assisted Navigation System (RFID-ANS) for VANETs. RFID-ANS consists of RFID readers installed on vehicles and passive RFID tags deployed on roads. As the maintenance for a passive tag is easy and its cost is less than a dollar, it is feasible to deploy a large number of passive tags for a relatively low cost over a broad area that is full of roadways.

Intuitively, RFID-ANS complements to the current GPS navigation system when GPS signals are not available (such as in tunnels) or if the GPS position is ambiguous to a vehicle (such as at cloverleaf intersections). But in practice, GPS does not provide sufficient information for navigation due to its low positioning accuracy (5 to 7 meters). Moreover, even combined with map-matching technologies, GPS still cannot achieve lane level positioning and cannot provide information regarding the traffic direction in the current lane. Nevertheless, these information are necessary to prevent vehicles from entering a wrong way when roads are under construction or lanes are temporarily borrowed by the traffic along a different direction. Our RFID-ANS is designed to address such problems. Its convenience and benefits give incentives for users to install

- W. Cheng and X. Cheng are with the Department of Computer Science, The George Washington University, Washington, DC 20052.

E-mail: wcheng@gwmail.gwu.edu, cheng@gwu.edu.

- M. Song is with the Department of Electrical and Computer Engineering, Old Dominion University, Norfolk, VA 23529. E-mail: msong@odu.edu.

- B. Chen is with the Department of Computer Information Science, University of Macau, Macau, PR China. E-mail: bchen@umac.mo.

- W.W. Zhao is with the Cornell University, 801 22ND Street NW, Suite 704, Washington, DC 20052. E-mail:wenxizhao@yahoo.com.

Manuscript received 1 Feb. 2011; revised 18 Aug. 2011; accepted 30 Sept. 2011; published online 19 Oct. 2011.

Recommended for acceptance by C.-Z. Xu.

For information on obtaining reprints of this article, please send e-mail to: tpds@computer.org, and reference IEEECS Log Number TPDS-2011-02-0056. Digital Object Identifier no. 10.1109/TPDS.2011.259.
RFID readers on their vehicles. Additionally, RFID-ANS can be configured to provide electrical traffic signs. It might be essential to future autonomous vehicle systems as this system can provide more precise real time road information for traffic scheduling. Note that the RFID reader attached at a vehicle is independent of the vehicle model, and it can be easily upgraded to guide driving. Therefore, RFID-ANS could play an important role in the future complex driving environment that contains autonomous, semiautonomous, and man-controlled vehicles.

RFID-ANS is a ground navigation system that is designed for the lane level navigation. The issues relevant to a practical RFID-ANS in a complex vehicular environment have never been addressed before. To our knowledge, this is the first work that provides a systematic approach to designing a RFID-ANS. Our multifaceted contributions are stated as follows:

- We provide an analysis on the design criteria of RFID-ANS. These criteria serve as guidelines for the design of the RFID readers and the deployment of the RFID tags. We present the relationships among these design criteria, and investigate how they should be used cooperatively to achieve the objectives of the navigation system. Based on these criteria, we identify the parameters that are important for the RFID-ANS design.

- We present the design of the RFID readers for RFIDANS in detail. The ranges of the critical parameters for the RFID readers are derived according to the requirements of the navigation system and the tag deployment.

- We jointly consider the design of the RFID reader's read interval and the deployment of the RFID tags, such that the cost and energy consumption can be optimized as long as the requirements of the navigation system are satisfied. The proposed methods for read attempt scheduling and tag deployment are robust and adaptable to dynamic road environments. 
- We propose methods to estimate the vehicle position. The accuracy of the estimated position and the performance of the designed RFID-ANS are analyzed.

The rest of the paper is organized as follows: Section 2 summarizes the most related work to our research. In Section 3, our RFID-ANS model is presented. The design criteria and their relationships, and the set of important parameters for RFID-ANS design and deployment, are introduced in this section as well. We detail the design of the RFID reader in Section 4. The deployment strategy of the tags is proposed in Section 5. In Section 6, we introduce two vehicle position estimation methods. Section 7 illustrates an example RFID-ANS with practical settings. Finally, this paper is concluded in Section 9.

\section{Related Work}

A RFID system is composed of RFID tags and RFID readers. A RFID tag stores data, and a RFID reader accesses the tag to collect the data through wireless communications. There exist two types of RFID tags: active tags, which contain power modules to support wireless communications, and passive tags, which power their transmissions through the energy absorbed from the radio waves of the RFID readers. Compared to active RFID tags, passive RFID tags are easier to maintain as they do not need power, and their cost can be as low as several cents. Therefore, passive RFID tags are more appropriate for applications that require a large number of tags.

Traditionally, RFID tags were designed for commercial applications to replace the bar codes for asset counting [1], [2] and identification [3]. One important challenge in such applications is how to handle the read collision problem that occurs when one or more RFID readers query multiple RFID tags roughly simultaneously in a small area. As a result, most existing research focuses on anticollision protocol design to schedule the reader's read requests and the tag's responses [4], [5], [6]. In RFID-ANS, read collision is not possible as our design guarantees the one-to-one coupling of a RFID reader and a tag in a restricted area.

RFID systems have been deployed for VANETs, in which RFID tags are installed on vehicles while RFID readers are deployed on stationary infrastructures. For example, in a typical Electronic Toll Collection (ETC) system [7], automatic toll RFID readers are installed at the gate. A RFID tag (attached to the E-ZPass on a vehicle) is read by the reader when a vehicle passes by the gateway. The toll system identifies the vehicle through the data obtained from the RFID tag, and automatically charges to the vehicle's or the driver's account. A similar system is established for parking fee collection in [8]. Compared to these systems, RFID-ANS contains stationary tags on roads while readers move with vehicles at high speeds.

The most related work to RFID-ANS are reported in [9], [10], [11]. Chon et al. [9] propose the idea of using stationary RFID tags deployed on roads to localize vehicles when passing by. The feasibility of utilizing RFID tags for navigation when vehicles move at high speeds is investigated through an experiment in which a RFID reader reads the data in a tag when the tag is dropped down to the ground. Lee et al. [10] study the relationship between the tag read latency and the vehicle's speed, and evaluates their results on a test road. These two works demonstrate the feasibility and practicality of applying commercial RFID tags and readers in the vehicular environment. But none of them considers critical issues such as tag deployment and read scheduling, which are important to the design of a practical RFID-ANS as they mainly focus on the concept and feasibility study. In the Road Beacon System proposed in [11], RFID tags serving as traffic signs are deployed in the pavement and vehicles get the road information through reading the tags. The technical details of this work are unavailable to our best knowledge.

\section{RFid Assisted Navigation System Model}

In our RFID-ANS model, passive RFID tags are deployed at the centers of the lanes and a RFID reader's antenna is installed at the center of a vehicle's front bumper, since this position exhibits the minimum error rate [10].

We assume that a RFID tag can provide its physical position, the lane's current traffic direction, and the road's name, which can help the vehicle localize itself at a cloverleaf intersection or in a tunnel. A moving vehicle can obtain its current position through reading the tags when passing by. According to [10], a typical RFID reader's read area is a function of its antenna's height, read angle, and pitch angle. The details on how to compute the read area of a RFID reader are given in Section 1 of the supplementary file, which can be found on the Computer Society Digital Library at http://doi.ieeecomputersociety. org/10.1109/TPDS.2011.259.

The design criteria of our RFID-ANS are explained as follows:

1. Each RFID tag should be covered by no more than one RFID reader's read area at any instant of time.

2. Each RFID reader's read area should cover no more than one tag at any instant of time.

3. If a vehicle is in a lane, the vehicle should be able to read tags that are deployed in the lane.

4. If a vehicle can read a tag, at least half of its body should be in the lane where the tag is deployed.

5. If less than half of a vehicle is in a lane, the vehicle should not be able to read any tag in the lane.

6. RFID tags should be deployed according to the road navigation requirements. In our study, navigation requirements are described by where and when a vehicle should successfully read tags.

7. A vehicle should schedule its read attempts such that the road navigation requirements can be satisfied and energy can be conserved.

Unlike traditional RFID applications, in which a RFID reader might encounter multiple tags at the same time, we require a one-to-one coupling of readers and tags at any instant of time in a RFID-ANS, as stated by the first two design criteria. In VANETs, any two moving vehicles cannot stay at the same position simultaneously. Thus, any two vehicles should not contact the same RFID tag at the same time. This means that a RFID tag should only be 
TABLE 1

The Parameters for a RFID-ANS

\begin{tabular}{|l|l|}
\hline$L_{\text {read }}$ & The RFID reader's read length \\
\hline$W_{\text {read }}$ & The RFID reader's read width \\
\hline$W_{\text {lane }}$ & The lane width \\
\hline$L_{V \min }$ & The minimum vehicle length \\
\hline$W_{V \min }$ & The minimum vehicle width \\
\hline $\bar{V}$ & The road speed limit \\
\hline$D_{\text {tag }}$ & The distance between two consecutive RFID tags \\
\hline$S_{\text {data }}$ & The RFID tag's data size \\
\hline$R_{\text {tag }}$ & The RFID tag's data transmission rate \\
\hline
\end{tabular}

read by at most one vehicle at any instant of time. On the other hand, a vehicle cannot appear at two different positions at the same time. Therefore, a vehicle's read area should cover at most one tag at any instant of time. In RFIDANS, this one-to-one coupling guarantees that transmission collisions can be avoided and the tag's random access time, which is employed to avoid collisions in traditional RFID applications, can be eliminated. As a result, the full theoretical RFID reader's read area, as calculated in [10], can be utilized by a vehicle to read tags. According to the experimental results in [10], a RFID reader's effective read length is about 60 percent of its theoretical value in VANETs due to the long random access time of its tags. As a result, the maximal vehicle's velocity for reliably reading a RFID tag is limited to $60 \mathrm{mph}$ (100 kph) [9]. In our design, the first two criteria theoretically eliminate the necessity of the RFID tag's random access time. Therefore, the effective read length can be maximized, and the read success rate can be improved for high speed VANETs.

The third, fourth, and fifth design criteria regulate the RFID reader's read width to achieve the lane level navigation. These three criteria guarantee that a tag can be read by vehicles in the same lane, but not be read by vehicles at different lanes.

To satisfy the navigation requirements, the sixth design criterion is proposed to guide the deployment of RFID tags. To facilitate the cooperation among deployed tags, the seventh design criterion is proposed, which requires a RFID reader to smartly schedule its read attempts such that it can successfully read tags to support its navigation, and the energy can be saved from avoiding unnecessary read attempts.

In summary, the first, second, and third criteria are proposed for the RFID read length design; the first five criteria join together to guide the design of the RFID read width; the sixth criterion is for RFID tag deployment; and the first, second, and seventh criteria are needed to facilitate the cooperation between tag deployment and reader design.

The most significant parameters for a RFID-ANS are defined in Table 1. We analyze the relationship among these parameters and discuss the relevant issues in the following two sections.

\section{RFID Reader Design}

In this section, we analyze the settings of the critical parameters for RFID readers to meet the RFID-ANS design criteria.

\subsection{RFID Reader's Read Length}

In RFID-ANS, the reader's read length should be carefully designed in order to successfully read the tags' information, as indicated by the first three design criteria. The RFID reader's read length is bounded by (1), where $\delta$ is the read length loss ratio. The derivation of (1) is reported in the supplementary file, available online.

$$
\frac{\bar{V} \times S_{\text {data }}}{R_{\text {tag }} \times \delta}<L_{\text {read }}<\min \left\{D_{\text {tag }}, L_{\text {Vmin }}\right\} .
$$

\subsection{RFID Reader's Read Width}

As mentioned in Section 3, the RFID reader's read width should be designed to satisfy the first five design criteria. The RFID reader's read width is bounded by (2). The derivation of (2) is reported in the supplementary file, available online.

$$
W_{\text {lane }}-W_{\text {Vmin }}<W_{\text {read }}<W_{\text {lane }} .
$$

\subsection{Considerations for Lane Change}

The fourth and fifth design criteria can be satisfied by (2) when the vehicle changes its lane smoothly. When a vehicle changes its lane sharply, it could read the tag deployed in the target lane where less than half of its body is present. This phenomenon contradicts the fourth and fifth design criteria. However, as the target lane is the one that the vehicle is heading for and the vehicle will present shortly, we can tolerant such a reasonable exception in our RFID-ANS design. Then, the problems that should be considered for the case of a sharp lane change include: 1 ) the vehicle should not be able to reach two tags simultaneously; 2) the vehicle should not be able to reach the tag deployed in the lane that it is leaving when most of its body has left the lane; and 3) a RFID tag should not be reached by more than one vehicle.

To address the first problem, we require that the distance between two consecutive RFID tags to be lower bounded by the reader's read area, as shown in (3). Moreover, the bounds of the read length defined by (1) is rewritten as (4). For details on the derivation of these equations please see the supplementary file, available online.

$$
\begin{gathered}
D_{\text {tag }}^{2}>W_{\text {read }}^{2}+L_{\text {read }}^{2}, \\
\frac{\bar{V} \times S_{\text {data }}}{R_{\text {tag }} \times \delta}<L_{\text {read }}<L_{V \text { min }} .
\end{gathered}
$$

Theorem 4.1 guarantees that the second problem can be resolved. A rigorous proof of this theorem, which summarizes our solution approach to the second problem based on the RFID reader design, is provided by the supplementary file, available online.

Theorem 4.1. The vehicle cannot read a RFID tag deployed in the lane that the most part of its body has left.

To address the third problem, we require the reader to stop reading when the vehicle is fully stopped. The details can be found in the supplementary file, available online.

Note that during a lane change, a vehicle might miss two tags when it makes an extreme sharp turn. This read miss 
can be tolerable because extreme sharp turns are abnormal and infrequent in reality. Moreover, one of the missed tags resides in the lane that the vehicle is leaving, and the vehicle will not miss the second tag in the lane that it is heading for.

\subsection{Adaptive Scheduling of the RFID Reader's Read Attempts}

In this section, we propose approaches to adaptively scheduling a RFID reader's read attempts such that the vehicle can read the RFID tags with a high success rate. Assume that tags have been deployed based on the road navigation requirements. A straightforward and effective scheduling method is to keep sending read attempts. However, when tags are sparsely deployed, the drawbacks of this approach are obvious because of its low success rate and high energy waste resulted from unnecessary read attempts. Although the energy consumption of making read attempts is not comparable to that of the vehicle alternator, the accumulative power waste from failed readings of all vehicles could be a large value that should not be ignored. Moreover, in the scenario where multivehicles are heading for the same lane or on a curved road, the probability of a read collision is large if all vehicles employ this straightforward method. Therefore, an ideal read scheduling method should be able to estimate the distance between two consecutive tags and be adaptable to different road segments where the distances between two tags might vary.

Generally speaking, read scheduling seeks to determine when RFID readers should send read attempts. According to Section 4.3, vehicles should not attempt to read tags when they are completely stopped. Moreover, they should reschedule their readings after they change lanes or enter new road segments. We assume that a lane change can be detected through monitoring wheel revolutions; and the system can be aware of the event via digital maps that a vehicle is entering a new road. Therefore, we focus on scheduling the read attempts when a vehicle stays in its lane. We start from a time at which a read attempt reaches a tag and results in a successful data read operation.

A vehicle is aware of it's RFID reader's setting, therefore it can calculate the length of a tag's successful read area, as illustrated by the shadowed area shown in Fig. 1a. The vehicle can successfully obtain the data from a tag if it schedules its read attempt in the successful read area. The length of the successful read area, $L_{\text {success }}$, is calculated by (5), where $L_{\text {min }}^{\prime}$ is the minimum read length determined by the current vehicle speed. Note that $L_{\min }^{\prime}$ increases with the vehicle's speed, as a result $L_{\text {success }}$ becomes smaller if the vehicle is speeding up given $L_{\text {read }}$. Thus, it is believable that a speeding vehicle would slow down to get a larger chance of successfully utilizing the RFID-ANS service. The computation of $L_{\min }^{\prime}$ is detailed in the supplementary file, available online.

$$
L_{\text {success }}=L_{\text {read }}-L_{\text {min }}^{\prime} .
$$

In order to successfully obtain the data, we should consider how to schedule a vehicle's read attempts at the tag's successful read area. Generally speaking, the vehicle should send a read attempt when traveling a distance of $D_{t a g}$ after a successful read attempt. Then, the key question

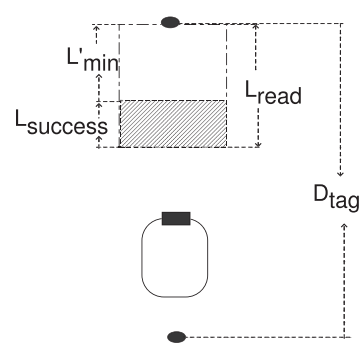

(a)

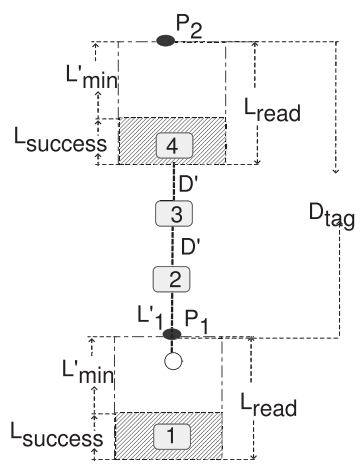

(b)
Fig. 1. (a) The successful read area to obtain a RFID tag's data. (b) The initial read attempt happens in the tag's successful read area. Here, rounded rectangles are the positions at which the vehicle should send the read attempts, $P_{1}$ and $P_{2}$ are the tags' positions, and the white circle is the position at which the vehicle finishes its initial read attempt.

is how can the vehicle obtain the distance between two consecutive tags. A straightforward method is for the tag to store the distance to the next tag. Obviously, this method is hard to implement and maintain. Furthermore, it is not necessary and is detrimental to RFID-ANS because the tag's increased data size results in a longer data transmission time and a smaller successful read area, according to (5). Therefore, in our RFID-ANS design, we assume that a tag does not have the distance information. Next, we focus on how vehicles should schedule their read attempts and how to obtain the $D_{t a g}$ value.

Note that a vehicle can measure the distance between any two positions it has passed as it knows its current speed and time. Also note that we assume that a lane change can be detected through monitoring wheel revolutions, and that the vehicle can detect whether it is entering a new road or not via digital maps. Then, the vehicle should always initiate a read attempt when it starts, enters a new road, or changes its lane. Its initial read attempt falls into one of the following three possibilities.

1. Successfully obtains a tag's data.

2. Successfully reaches a tag, but fails to obtain the data.

3. Fails to reach a tag.

Case 1. If the initial read attempt obtains a tag's data, it happens in the tag's successful read area. Then, the schedule of the following read attempts should follow Fig. 1b, with

$$
L_{1}^{\prime}=\sqrt{W_{\text {read }}^{2}+L_{\text {read }}^{2}}-L_{\text {read }}
$$

and $D^{\prime}$ is close to but smaller than $L_{\text {success }}$. According to (3), $D_{\text {tag }}>L_{1}^{\prime}+L_{\text {read }}$. Then, after obtaining the position of the first tag $P_{1}$ at the white circle, the vehicle should send the second read attempt after a distance of $L_{1}^{\prime}$. As the vehicle passes the white circle before $P_{1}$, the distance between the white circle and the next tag must be larger

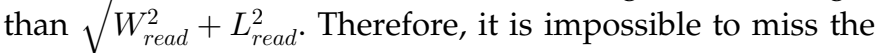
next tag if the second read attempt is scheduled after $L_{1}^{\prime}$. After that, the following read attempts are scheduled for 


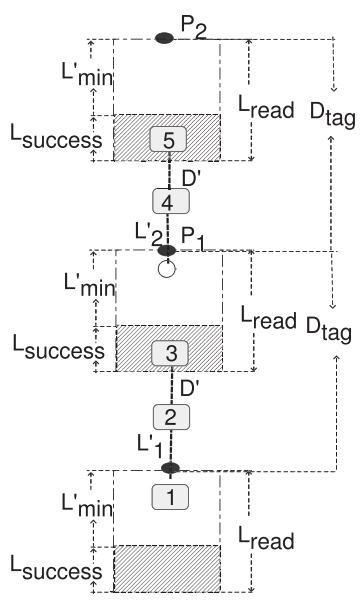

(a)

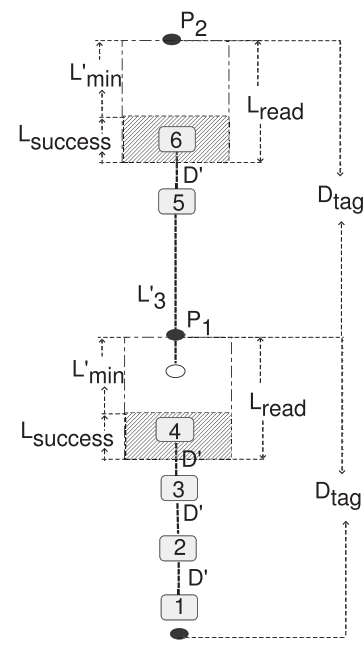

(b)
Fig. 2. (a) The initial read attempt happens in the tag's $L_{\min }^{\prime}$ area. (b) The initial read attempt happens at the blank area.

every distance $D^{\prime}$ until the next tag has been successfully read. Note that $D^{\prime}$ could be any number that is slightly smaller than $L_{\text {success }}$, which should be calculated based on the vehicle's current speed. As $D^{\prime}<\approx L_{\text {success, }}$ the scheduled read attempts must be able to read the tag $P_{2}$. Then, the distance between two consecutive tags can be estimated by $\left|P_{2}-P_{1}\right|$.

In this case, as the second read attempt is scheduled after $L_{1}^{\prime}$, it is possible that $P_{1}$ can be successfully read or reached by several read attempts when $W_{\text {read }}$ is small and $L_{\text {success }}$ is large. We ignore the results of these read attempts because there is no tag between $P_{1}$ and $P_{2}$.

Case 2. If the initial read attempt successfully reaches a tag but fails to obtain the data because of insufficient contact time, the vehicle was in the tag's $L_{\text {min }}^{\prime}$ area when it sent the read attempt. Then, the following read attempts can be scheduled as illustrated in Fig. 2a.

The second read attempt is scheduled at $L_{1}^{\prime}$ after the initial read attempt, where $L_{1}^{\prime}$ is defined in case 1 . As the position of the first tag is higher than the vehicle's first attempt position in Fig. 2a, it is not necessary to schedule any read attempt for the tag $P_{1}$ before $L_{1}^{\prime}$ for the same reason as in the first case. After the second read attempt, the following attempts are scheduled in every $D^{\prime}$ distance until the tag $P_{1}$ is successfully read. When $P_{1}$ is obtained at the white circle, the vehicle sends the next read attempt after a distance of $L_{2}^{\prime}$, where $L_{2}^{\prime}=\max \left(D_{\text {miss }}-L_{\text {min }}^{\prime}, D^{\prime}\right)$, and $D_{\text {miss }}$ is the distance between the vehicle's first attempt position and the most recent attempt position at which it fails to reach the tag $P_{1}$. In this example, $D_{\text {miss }}$ equals the distance between the vehicle's first and second attempt positions. Furthermore, it is not necessary to schedule a read attempt before $L_{2}^{\prime}$ to read the tag $P_{2}$. After this attempt, the following read attempts are made for every distance $D^{\prime}$ until the tag $P_{2}$ is successfully read. When $P_{1}$ and $P_{2}$ are available, they can be used to estimate $D_{\text {tag }}$.

Case 3. If the initial read attempt fails to reach a tag, it happens in a blank area. The following read attempts are scheduled according to Fig. 2b. At the beginning, the vehicle sends attempts every distance $D^{\prime}$ until the tag $P_{1}$ is

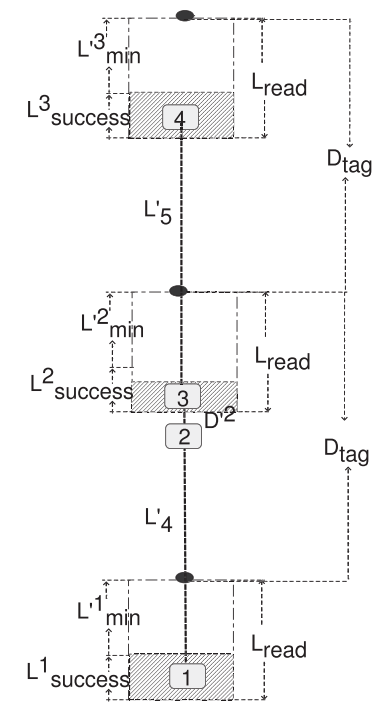

(a)

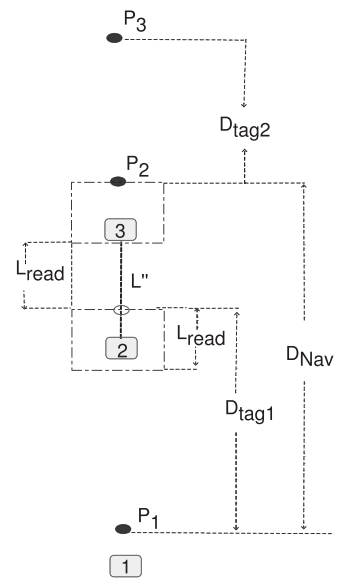

(b)
Fig. 3. (a) The scheduling of read attempts. (b) The deployment strategy of RFIF tags.

successfully read. After obtaining the tag $P_{1}^{\prime}$ s information at the white circle, the next read attempt is made after a distance of $L_{3}^{\prime}=D_{\text {miss }}+D^{\prime}$, where $D_{\text {miss }}$ is defined as before. In the example, $L_{3}^{\prime}$ equals the distance from the vehicle's first attempt position to its fourth attempt position. As the vehicle's first attempt position is above the lowest tag, and the white circle is below $P_{1}$, it is not necessary to schedule any read attempt before $L_{3}^{\prime}$. After this, the following read attempts are scheduled for every distance of $D^{\prime}$ until tag $P_{2}$ is successfully read. Note that the above three read schedulings for $D_{t a g}$ estimation are robust to the vehicle's velocity variation because $D^{\prime}$ is calculated based on the current speed.

As the vehicle's speed is not always constant, the boundary of the successful read area might vary. In addition, the vehicle does not know exactly when its front bumper passes a tag. Therefore, simply scheduling the read attempts for every $D_{t a g}$ might not result in a successful obtention of the data when the vehicle is accelerating. To address this challenge, the vehicle schedules its read attempts as illustrated by Fig. 3a after acquiring the distance between two consecutive tags, where $L_{4}^{\prime}=D_{\text {tag }}-L_{\text {success }}^{1}+D_{2}^{\prime}, L_{5}^{\prime}=D_{\text {tag }}-L_{\text {success }}^{2}+D_{3}^{\prime}$.

In summary, the vehicle should send the next read attempt after a distance $L^{\prime}$ from its most recent attempt position at which a success read attempt is made. $L^{\prime}$ is defined in

$$
L^{\prime}=D_{\text {tag }}-L_{\text {success }}^{\text {previour }}+D_{\text {current }}^{\prime},
$$

where $L_{\text {success }}^{\text {previour }}$ is the length of the successful read area of the vehicle's most recent successful read, and $D_{\text {current }}^{\prime}$ is close to and smaller than the length of the current successful read area based on the vehicle's velocity. If this scheduled read attempt falls in the blank area, the vehicle keeps sending attempts every $D_{\text {current }}^{\prime}$ until the next tag's information is obtained before it travels distance $D_{t a g}$, as illustrated by Fig. 3a. We present the read scheduling method to address the $D_{\text {tag }}$ 's change in the next section. 


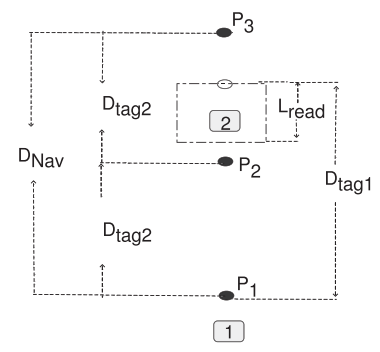

(a)

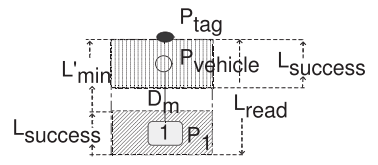

(b)
Fig. 4. (a) The tag $P_{2}$ is missed by the vehicle. Here, $D_{t a g 1}$ is the old distance, $D_{t a g 2}$ is the new distance, $D_{t a g 2}<D_{t a g 1}$, and the white ellipse is the estimated position for the tag $P_{2}$ based on $D_{t a g 1}$. (b) The vehicle's position estimation. Here, $P_{1}$ is the vehicle's position at which it sends the read attempt, $D_{m}$ is the distance measured from $P_{1}$ to the vehicle's current position, and the shaded area in the top half of the figure is the area where a success read could finish.

\section{RFid Tag Deployment Strategy}

Assume that the navigation system requires a vehicle to obtain its position through reading RFID tags once in every distance $D_{A N S}$. Note that this distance might vary with different road segments. For example, the distance required in urban areas might be much smaller than the one required for rural areas, and the distance required in the areas near intersections or exits should be smaller than the one required in normal road segments. Thus, the tags' deployment is not a trivial task. The strategy for deploying RFID tags should satisfy different navigation requirements while guarantee that a vehicle can keep the required level of navigation running seamlessly.

As mentioned in Section 4.4, vehicles in RFID-ANS always reschedule their read requests by initiating a read attempt when they have changed lanes or entered new road segments. Note that we label a road segment as new when the vehicle passes an intersection or takes an exit. Then, we focus on the deployment strategy of RFID tags in continuous road segments where the navigation requirements vary. In continuous road segments, we require that a vehicle reschedule its read attempts when it has failed to contact the tag at an estimated position or failed to obtain the tag's data.

Generally speaking, the change of the navigation requirements falls into one of the following two categories:

1. The required distance changes to a smaller value.

2. The required distance changes to a larger value.

Category 1. If the tag is simply deployed according to the changed distance as shown in Fig. 4a, the vehicle might miss $P_{2}$. As the vehicle schedules its read attempts based on the previous estimated distance $D_{t a g 1}$ from (6), the distance between two scheduled consecutive read attempts is larger than $D_{t a g 2}$. As a result, the vehicle passes the tag $P_{2}$ without reading it. Then, after the vehicle obtains the next $\operatorname{tag} P_{3}$ 's data, the distance between two success reads is $D_{\text {Nav }}=2 \times D_{t a g 2}$, which is larger than any of $D_{t a g 2}$ and $D_{t a g 1}$. Therefore, the navigation requirements might not be satisfied between $P_{1}$ and $P_{3}$.

Note that $D_{\text {Nav }}$ determines the system's real-time navigation accuracy. We give it a special name navigation precision in this paper. To achieve seamless navigation when the required distance is decreasing, we propose the following deployment strategy as illustrated in Fig. 3b:
The distance between $P_{1}$ and $P_{2}$ is $D_{\text {Nav }}=D_{t a g 1}+2 \times L_{\text {read }}$, and $L^{\prime \prime}=L_{\text {read }}+L_{\text {success }}$ is the distance after which the vehicle should reinitiate read attempts since it notices that the next tag is not at the estimated position after traveling distance $D_{t a g 1}$ without reaching any tag.

It can be verified that the vehicle can obtain the data of the tag $P_{2}$ using the deployment strategy mentioned above. Then, to guarantee seamless navigation, we require $D_{N a v}<D_{A N S}$. This results in a general deployment requirement shown in

$$
D_{\text {tag }}<D_{\text {ANS }}-2 \times L_{\text {read }} .
$$

According to (3), (4), and (7), we obtain

$$
\sqrt{W_{\text {read }}^{2}+L_{\text {read }}^{2}}<D_{\text {tag }}<D_{A N S}-2 \times L_{V \text { min }} \text {. }
$$

Category 2. For the second category, we need to simply increase $D_{\text {tag }}$ between two consecutively deployed tags. As the vehicle automatically reschedules its read attempts after $L^{\prime \prime}$ if it fails to read or reach the next tag, in order to guarantee that the vehicle can obtain the next tag's data, we require that the difference between the old, short deployment distance and the new, long deployment distance be larger than $2 \times L_{V \min }$. Then, the vehicle should not miss any deployed tags. As a result, the vehicle can obtain the required level of seamless navigation.

Based on the tag deployment strategy presented above, a vehicle can smoothly adapt to varied navigation requirements without a bitch. As a result, the real-time navigation precision of the designed RFID-ANS is bounded by the minimum navigation requirements. According to (8), the minimum $D_{A N S}$, is $3 \times L_{V \min }+W_{\text {lane, }}$ while $W_{\text {lane }}>W_{\text {read }}$ and $L_{V \min }>L_{\text {read }}$.

\section{Vehicle Position Estimation}

We assume that a vehicle can estimate its position as soon as it successfully reads a tag. As mentioned above, the vehicle does not know the exact time at which its front bumper passes a tag even if it can obtain the tag's position $P_{t a g}$. Therefore, as shown in Fig. $4 \mathrm{~b}$, the vehicle should not simply use $P_{t a g}$ as its current position $P_{\text {vehicle }}$. We use (9) to estimate a vehicle's position. The position error is bounded by $\frac{L_{\text {success }}}{2}$, which is less than half of the lane width. Therefore, RFID-ANS can achieve lane level navigation. For a detailed discussion about the vehicle position estimation, please see the supplementary file, available online.

$$
P_{\text {vehicle }}=\left|P_{\text {tag }}-\frac{L_{\text {success }}}{2}\right| \text {. }
$$

\section{A RFID-ANS EXAMPLE}

We setup an example RFID-ANS according to the standard for interstate highways in the United States. In our example, the data size of a RFID tag is set to 64 bits, and EM4222 chip [10] is employed as the reader. We assume that the navigation system requires the vehicle to successfully read a RFID tag once every 60 feet $(18.29 \mathrm{~m})$. Based on our analysis, this example RFID-ANS has a navigation precision of 38.4 feet $(11.7 \mathrm{~m})$, which satisfies the navigation system requirements. For details on this example, please see the supplementary file, available online. 


\section{Simulation}

In this section, we report our simulation results obtained from the example RFID-ANS mentioned in Section 7. We use two settings for $D_{t a g}$. One thousand tags are placed in a straight line, where $D_{t a g}$ is changed alternatively once every 50 tags. We add a tag deployment error to each tag, which represents the shift from the tag's real position to its expected position. A virtual vehicle is employed in our simulation study to test the performances of the proposed RFIF-ANS. In this study, we set $D^{\prime}=0.9 \times L_{\text {success. }}$. The results indicate that more than 97 percent of the deployed tags can be successfully read by the vehicle, that almost 80 percent of the scheduled read attempts can yield successful reads, and that the position error is always upper bounded by 2 feet. The details on our simulation results are reported in the supplementary file, available online.

\section{Conclusion}

We summarize our investigation on the design of RFIDANS in this section. The proposed RFID-ANS can achieve a navigation precision of $3 \times L_{V \min }+W_{\text {lane }}$ and the accuracy of the vehicle's estimated position is $L_{\text {success }}$. An ideal RFID tag for RFID-ANS should have a near zero access time. The important parameters for the design of the RFID reader and the deployment of the RFID tags should satisfy the following conditions:

$$
\begin{array}{r}
W_{\text {lane }}-W_{\text {Vmin }}<W_{\text {read }}<W_{\text {lane }} \\
W_{\text {read }}^{2}+L_{\text {read }}^{2}<W_{\text {lane }}^{2} \\
\frac{\bar{V} \times S_{\text {data }}<L_{\text {read }}<L_{\text {Vmin }}}{R_{\text {tag }} \times \delta} \\
\sqrt{W_{\text {read }}^{2}+L_{\text {read }}^{2}}<D_{\text {tag }}<D_{A N S}-2 \times L_{V \text { min }} .
\end{array}
$$

Note that in the above conditions, the effect of the read noise and that of the communication delays are modeled by the read length loss ratio $\delta$. In our future research, we will treat the two negative effects separately. The communication delay will be controlled by tag design and reader design. The effect of the read noise will be estimated through modeling and analysis. We will also focus on studying multilane tag codevelopment strategies and the corresponding read scheduling methods such that the system's performance can be improved in complex environments.

\section{ACKNOWLEDGMENTS}

The research of Wei Cheng, Xiuzhen Cheng, and Biao Chen is supported in part by the 973 Program of China under grant 2011CB302800, and the US National Science Foundation (NSF) under grant CNS-0852673. The research of Min Song is supported in part by NSF CAREER Award CNS0644247 and NSF IPA Independent Research and Development (IR/D) Program. Any opinions, findings, and conclusions or recommendations expressed in this material are those of the author and do not necessarily reflect the views of the NSF.

\section{References}

[1] H. Han, B. Sheng, C.C. Tan, Q. Li, W. Mao, and S. Lu, “Counting Rfid Tags Efficiently and Anonymously," Proc. IEEE INFOCOM '10, Mar. 2010.
[2] M. Kodialam and T. Nandagopal, "Fast and Reliable Estimation Schemes in Rfid Systems," Proc. MobiCom '06, pp. 322-333, 2006.

[3] L. Xie, B. Sheng, C.C. Tan, Q. Li, and D. Chen, "Efficient Tag Identification in Mobile Rfid Systems," Proc. IEEE INFOCOM '10, Mar. 2010.

[4] B. Metcalfe, "Steady-State Analysis of a Slotted and Controlled Aloha System with Blocking," SIGCOMM Computer Comm. Rev., vol. 5, no. 1, pp. 24-31, 1975.

[5] S.-R. Lee, S.-D. Joo, and C.-W. Lee, "An Enhanced Dynamic Framed Slotted Aloha Algorithm for Rfid Tag Identification," Proc. the Second Ann. Int'l Conf. Mobile and Ubiquitous Systems: Networking and Services (MOBIQUITOUS '05), pp. 166-174, 2005.

[6] J. Myung and W. Lee, "Adaptive Splitting Protocols for Rfid Tag Collision Arbitration," Proc. Seventh ACM Int'l Symp. Mobile Ad Hoc Networking and Computing (MobiHoc '06), pp. 202-213, 2006.

[7] E-zpass, http://www.e-zpassny.com, 2010.

[8] Z. Pala and N. Inanc, "Smart Parking Applications Using Rfid Technology," Proc. First Ann. RFID Eurasia, 2007.

[9] H.D. Chon, S. Jun, H. Jung, and S.W. An, "Using Rfid for Accurate Positioning," J. Global Positioning Systems, vol. 3, nos. 1/2, pp. 3239, 2004.

[10] E.-K. Lee, Y.M. Yoo, C.G. Park, M. Kim, and M. Gerla, "Installation and Evaluation of Rfid Readers on Moving Vehicles," Proc. ACM Sixth Int'l Workshop VehiculAr InterNETworking (VANET '09), pp. 99-108, Sept. 2009.

[11] Road Beacon System, http://www.roadbeacon.com/, 2010.

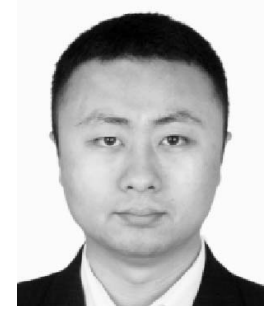

Wei Cheng received the $\mathrm{PhD}$ degree in computer science from the George Washington University, Washington, DC, in 2010 . $\mathrm{He}$ is a research scientist in the Department of Computer Science, the George Washington University. His research interests include RFID system on road, cyber physical systems, wireless networks, and polynomial-time algorithm design and analysis. He is a member of the IEEE and ACM.

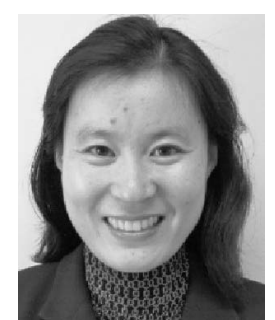

Xiuzhen Cheng received the MS and $\mathrm{PhD}$ degrees in computer science from the University of Minnesota, Twin Cities, in 2000 and 2002, respectively. She is an associate professor in the Department of Computer Science, The George Washington University, Washington, DC. Her current research interests include cyber physical systems, wireless and mobile computing, sensor networking, wireless and mobile security, and algorithm design and analysis. She worked as a program director for the US National Science Foundation (NSF) for six months in 2006 and joined the NSF again as a part-time program director in April 2008. She received the NSF CAREER Award in 2004. She is a senior member of the IEEE.

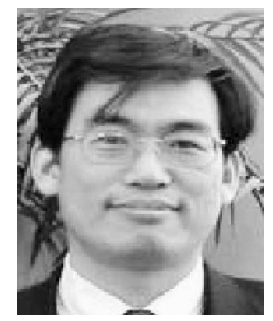

Min Song received the $\mathrm{PhD}$ degree in computer science from the University of Toledo in 2001. $\mathrm{He}$ is currently a program director in the Division of Computer and Network Systems, Directorate for Computer and Information Science and Engineering, US National Science Foundation (NSF). He is also an associate professor in the Department of Electrical and Computer Engineering at Old Dominion University. His research interests include design, analysis, and evaluation of cognitive radio networks, wireless sensor networks, wireless mesh networks, wireless local area networks, and mobile ad hoc networks. He joined Old Dominion University in June 2002. At ODU, he is the recipient of Excellence Research Award and has been awarded early tenure and promotion. He is the founder and director of the Wireless Networking @ ODU Laboratory. He is the recipient of NSF CAREER Award and the recipient of DOE GAANN Award. He is a senior member of the IEEE. 


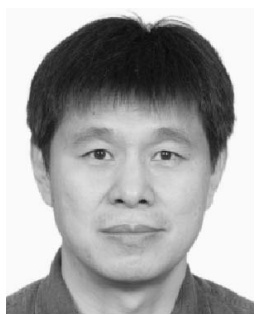

Biao Chen received the BS degree in computer science from Fudan University in China and the MS degree in mathematics and PhD degree in computer science from Texas A\&M University, respectively. After graduation, he joined the Department of Computer Science in University of Texas at Dallas as an assistant professor. Currently, he is a visiting professor in the Department of Computer and Information Science of University of Macau. His research interests include distributed systems, networking, and security. $\mathrm{He}$ is a member of the Sigma Xi, IEEE, and ACM.

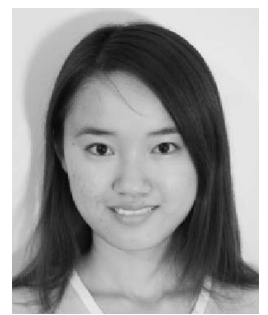

Wendy W. Zhao is an undergraduate student at Cornell University. She spent the summers of 2009 and 2010 as an intern in the Computer Science Department at the George Washington University under the guidance of Dr. Xiuzhen Cheng.

$\triangleright$ For more information on this or any other computing topic, please visit our Digital Library at www.computer.org/publications/dlib. 